\title{
Sex stratification of an inflammatory bowel disease genome search shows male-specific linkage to the HLA region of chromosome 6
}

\author{
Sheila A Fisher ${ }^{\star}, 1$, Jochen Hampe ${ }^{2}$, Andrew JS Macpherson ${ }^{3}$, Alastair Forbes ${ }^{4}$, \\ John E Lennard-Jones ${ }^{4}$, Stefan Schreiber ${ }^{2}$, Mark E Curran ${ }^{5}$, Christopher G Mathew ${ }^{1}$ and \\ Cathryn M Lewis ${ }^{1}$
}

\begin{abstract}
${ }^{1}$ Division of Medical and Molecular Genetics, Guy's, King's and St Thomas' School of Medicine, King's College London, UK; ${ }^{2}$ Department of General Internal Medicine, University Hospital Kiel, Christian-Albrechts-University, Kiel, Germany; ${ }^{3}$ Division of Medicine, Guy's, King's and St Thomas' School of Medicine, King's College London, UK; ${ }^{4}$ St Mark's Hospital, Harrow, UK; ${ }^{5}$ DNA Sciences, Fremont, California, USA
\end{abstract}

Inflammatory bowel disease (IBD) is a multifactorial disorder, with both genetic and environmental factors contributing to the two clinical phenotypes of Crohn's disease (CD) and ulcerative colitis (UC). The underlying genetic model is thought to involve multiple genes with complex interactions between disease loci, and the NOD2 gene on chromosome 16 has recently been identified as a CD susceptibility locus. Several genome-wide linkage studies have identified candidate regions, but there has been little replication across studies. Here we investigate the role of sex-specific loci in susceptibility to IBD. Linkage data from our previously reported genome search and follow-up study were stratified by the sex of the affected sib pair. Non-parametric linkage analysis was performed using Genehunter Plus. Simulation studies were used to assess the significance of differences in LOD scores between male and female families for each chromosome. Several regions of sex-specific linkage were identified, including existing and novel candidate loci. The major histocompatibility region on chromosome $6 \mathrm{p}$, referred to as IBD3, showed evidence of male-specific linkage with a maximum LOD score of 5.9 in both CD and UC male-affected families. Regions on chromosomes 11, 14 and 18 showed strong evidence of linkage in male-affected families but not in female-affected families. No evidence of sex-specific linkage was found in the IBD1 or IBD2 candidate regions of chromosomes 16 and 12 . The existence of sex-specific linkage is further evidence of the complex mechanisms involved in IBD and will facilitate future studies to identify susceptibility genes.

European Journal of Human Genetics (2002) 10, 259-265. DOI: 10.1038/sj/ejhg/5200792

Keywords: linkage; stratification; chromosome 6; HLA

Introduction

The genetic component of inflammatory bowel disease (IBD) includes a complex pattern of inheritance, with multiple

${ }^{*}$ Correspondence: SA Fisher, Division of Medical and Molecular Genetics, GKT School of Medicine, King's College London, 8th Floor Guy's Tower, Guy's Hospital, London SE1 9RT, UK. Tel: +44 207955 2516;

Fax: +44 207955 4644; E-mail: sheila.fisher@kcl.ac.uk

Received 13 December 2001; revised 7 February 2002; accepted 7 February 2002 susceptibility genes thought to be involved. Recent studies have identified NOD2 on chromosome 16 as a susceptibility gene for Crohn's disease (CD). ${ }^{1,2,3}$ Genome-wide linkage studies to date have reported putative disease susceptibility loci on several chromosomes: candidate regions for inflammatory bowel disease loci have been identified on chromosomes $1,3,4,6,7,12,14$ and $16 .{ }^{4-8}$ Our previous genomewide linkage analysis confirmed linkage to chromosomes 16 (IBD1) and 12 (IBD2), and reported new suggestive evidence for linkage to chromosomes $1,4,6,10,22$ and $X .{ }^{9}$ Our follow- 
up study, with dense marker typing and an extended affected sib pair family collection, increased the evidence for linkage to chromosome $6 \mathrm{p}$ with a maximum multipoint LOD score of 4.2 at D6S461. ${ }^{10}$ This IBD3 linkage has been replicated in a Canadian population. ${ }^{11}$

Several studies have demonstrated the complex mechanisms involved in IBD, in particular different genetic inheritance models for Crohn's disease (CD) and ulcerative colitis (UC), or phenotypic heterogeneity. For example, a major susceptibility gene in the IBD1 region with a dominant inheritance for UC and a recessive inheritance for CD has been proposed; ${ }^{12}$ it has been suggested that the IBD2 locus makes a major contribution to UC susceptibility but plays a relatively minor role in susceptibility to $\mathrm{CD} .{ }^{13}$ Linkage and association have been demonstrated in clinical subgroups defined by age of onset and disease severity. ${ }^{14,15}$ Sex-specific differences in IBD are suggested by studies showing linkage to the $\mathrm{X}$ chromosome. ${ }^{9,16}$ However, there are few established differences in the prevalence of inflammatory bowel disease between males and females. ${ }^{17}$ This $\mathrm{X}$ chromosome IBD susceptibility locus may therefore be balanced by sex-specific autosomal loci, with the potential for locus heterogeneity and epistatic interactions. No previous IBD linkage studies have investigated this hypothesis, although in other immune-mediated diseases examples of sex-specific autosomal linkages exist. Differential evidence for linkage has been demonstrated in families with affected sib pairs of a particular sex, for example in Type I Diabetes, ${ }^{18}$ hypertension ${ }^{19}$ and osteoarthritis. $^{20}$

Genome-wide searches for linkage have been performed in many immune-mediated diseases, but results have not been easy to replicate across different studies. ${ }^{21}$ Disease genes of small effect are difficult to localise by linkage analysis, and stratification provides a method to increase the statistical power of a linkage study. In this paper, we re-analyse genome search data from inflammatory bowel disease, with stratification of families by the sex of affected individuals. Using the linkage data from our previously reported genome search ${ }^{9}$ and chromosome $6 \mathrm{p}$ follow-up study, ${ }^{10}$ families were stratified by the sex of affected individuals in each family to identify excess allele sharing specific to males or females. Simulation studies were used to assess the significance of chromosomal regions with an observed difference in evidence for linkage between male-only and female-only affected families.

\section{Subjects and methods}

As previously reported, 353 white northern European affected sibling pairs from 268 nuclear families were genotyped with 358 microsatellite markers across the genome. ${ }^{9}$ A further 75 affected sibling pairs were genotyped with an additional 11 markers in the region of linkage on chromosome $6,{ }^{10}$ which includes the HLA region. The general IBD phenotype was classified as Crohn's disease
(CD) or ulcerative colitis (UC); analyses were carried out for each of these phenotypic categories to identify loci specific to either of these sub-phenotypes. Family cohorts were recruited from a number of European centres. Informed, written consent was obtained from all study participants; recruitment protocols were approved by institutional review committees at each participating centre. Methods of family ascertainment, clinical phenotypes and genotyping are described in detail elsewhere. ${ }^{9,10}$

For each phenotype, nuclear families were stratified according to the sex of affected individuals within each family. Analyses were performed using all families (ALL), families with affected females only (F), and affected males only (M). Where a family contained both UC and CD affected siblings, linkage analysis for the CD phenotype classified UC affected individuals as having unknown affection status; similarly for CD cases in UC analysis. By this method, families with more than two affected offspring of mixed sex could be included in the analyses if a sibling pair were concordant for sex and phenotype. A summary of the number of families for each of the phenotypes (IBD, CD, UC), stratified by sex of affected siblings, is given in Table 1.

Multipoint linkage analysis was performed using Genehunter Plus, ${ }^{22}$ a modified version of the Genehunter program. $^{23}$ This program calculates a non-parametric LOD score using an identity-by-descent linear allele-sharing model. The 'ALL' scoring function was used, allowing allelesharing to be estimated from all affected individuals simultaneously. For each phenotype, genome-wide LOD scores were calculated for male and female affected families.

A simulation method was used to assess the difference in LOD scores between male and female families by chromosome, providing a test for heterogeneity between sex-specific affected families. The combined cohort of male-only and female-only families was randomly divided into two samples of size identical to male only/female only families for each phenotype. Each sample was analysed and the difference in maximum LOD score between the two groups for each chromosome was calculated. This was repeated 5000 times for a permutation test, and the proportion of simulations for which the observed result exceeded the simulated difference gives the probability of observing such a difference by chance.

Where sex-specific linkage was identified in CD families, the role of locus interaction with NOD2 was investigated by stratification of families by presence or absence of NOD2 mutations (3020insC, R702W, L1007P) in CD affecteds. ${ }^{1-3}$

\section{Results}

In the genome search family cohort, $60 \%$ of affected offspring were female. The distribution of IBD-affected sibpairs over male-only, female-only and mixed families differs significantly from the expected distribution, given this estimated male-female ratio and assuming random assort- 
Table 1 Overview of family cohorts stratified by sex of affected individuals

\begin{tabular}{|c|c|c|c|c|c|c|c|c|c|}
\hline \multirow[b]{2}{*}{ No. sibs } & \multicolumn{3}{|c|}{$C D$} & \multicolumn{3}{|c|}{$U C$} & \multicolumn{3}{|c|}{$I B D$} \\
\hline & ALL & $M$ & $F$ & ALL & $M$ & $F$ & ALL & $M$ & $F$ \\
\hline \multicolumn{10}{|c|}{ Genome search family cohort } \\
\hline 2 & 114 & 24 & 48 & 78 & 14 & 23 & 230 & 46 & 90 \\
\hline 4 & 1 & 0 & 0 & 0 & 0 & 0 & 3 & 0 & 0 \\
\hline Total & 119 & 26 & 52 & 90 & 18 & 25 & 268 & 52 & 98 \\
\hline 3 & 0 & 0 & 0 & 1 & 1 & 0 & 2 & 1 & 0 \\
\hline Total & 32 & 5 & 12 & 19 & 5 & 5 & 60 & 12 & 19 \\
\hline
\end{tabular}

ment of pairs ( $P=0.02$, chi-squared test). An excess of femaleonly families was observed for both CD and UC phenotypes. This excess was not unexpected, as females, and hence female affected sib-pairs, are easier to recruit as they are generally more willing to participate in epidemiological studies.

Genehunter Plus multipoint LOD scores for CD and UC phenotypes, from all families (ALL), and for the sub-groups of male only (M) and female only (F) affected families, are shown in Figure 1. Regions with a LOD score of at least 2 in either male or female families are shown in Table 2. Regions of linkage with the IBD phenotype are also listed. Eight regions demonstrated 'suggestive' evidence for linkage, as defined by a LOD score of 2.2 in a genome-wide search. ${ }^{24}$ Male-specific linkage was found in CD, UC and IBD phenotypes. Only two regions showed female-specific linkage, both for UC, despite the higher numbers of female-only families.

The highest LOD score for IBD was on chromosome $6 \mathrm{p} 21.3$ where linkage was identified in male affected families only (see Table 2). A difference between subgroups of comparable size was not observed in any of 5000 simulations. This malespecific linkage to chromosome 6 was also seen in CD affected sib pairs (Table 2), and in UC affected sib pairs (Male $\mathrm{LOD}=1.53$, Female $\mathrm{LOD}=0, P>0.05)$. The number of families in each subgroup is small for UC and the non-significant difference for UC affected sib pairs may reflect a lack of statistical power. This region corresponds to the previously identified linkage, ${ }^{9}$ and includes the HLA region. The dense marker typing on chromosome 6 with additional families increased the evidence for linkage in male only affected families (Figure 2). The maximum multipoint LOD score for IBD occurred at D6S291 (Male LOD=5.91, Female LOD=0.06, $P<0.0002)$, with evidence for linkage in both CD and UC phenotypes.

Simulations confirmed seven of the eleven other regions attaining a LOD score over 2 as sex-specific regions, with a significant difference in LOD between male and female families $(P<0.05)$. Two regions of linkage on chromosome $\mathrm{X}$ correspond to those identified previously from all families in our genome search. ${ }^{9}$ The linkage to IBD (Xp22, $\left.34 \mathrm{cM}\right)$ was identified in male affected families, whereas the second region of linkage (Xq24-26, $139 \mathrm{cM}$ ) was in female UC affected families only. Chromosome 1q showed linkage in male families only; this region of linkage is $55 \mathrm{cM}$ proximal to that previously reported without stratification by sex. ${ }^{9}$ Male-specific linkage was identified on chromosome 3 (IBD), corresponding to the previously identified candidate locus. ${ }^{5}$ The region of linkage in male families shown here on chromosome 14 is approximately $50 \mathrm{cM}$ distal from the putative locus on $14 \mathrm{q} 11 . .^{7,8}$ Other regions of male-specific linkage not previously identified as candidate regions included chromosome 11q21 and chromosome 18p11, which is more than $70 \mathrm{cM}$ proximal to the regions of linkage previously identified at 18q. ${ }^{7,8}$ Distinct male-specific linkage regions were identified on chromosome 11 for $\mathrm{CD}$ and UC phenotypes, separated by $64 \mathrm{cM}$, although the difference in LOD score between male and female families was not significant from simulations. The only autosomal region which showed suggestive linkage in female families was chromosome 20 (UC), although the evidence for a difference between male and female families was marginal $(P=0.054)$. No interesting regions were observed in female CD or IBD families. The established IBD candidate loci on chromosome 16 (IBD1) and 12 (IBD2) did not exhibit sex-specific linkage.

The male and female specific LOD scores for chromosome 22 were both greater than the overall LOD score for all families (Male $\mathrm{LOD}=1.38$, Female $\mathrm{LOD}=2.29$, $\mathrm{ALL} L O D=1.28$ ) at $32 \mathrm{cM}$ for IBD. The combined LOD score for male only and female only IBD affected families was 3.66, suggesting that there may be two sex-specific disease loci in this region. A simulation study was used to assess this LOD score for significance. A sample of 149 families (equivalent to 50 male only and 99 female only IBD families) was randomly selected from the ALL family cohort and the maximum LOD score recorded. In 5000 simulations, a maximum LOD score of at least 3.66 was observed only five times $(P=0.001)$.

NOD2 has been confirmed as a CD susceptibility locus. CD families were therefore stratified by presence or absence of NOD2 mutations, and regions showing sex-specific linkage in $\mathrm{CD}$ families were re-analysed. The greatest difference in 


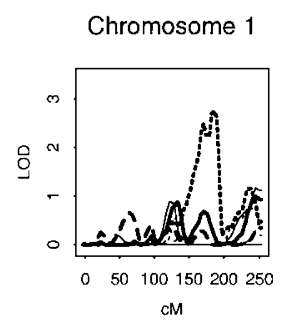

Chromosome 7

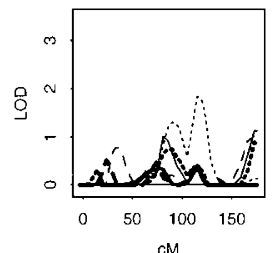

Chromosome 13

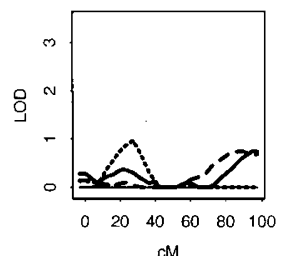

$\mathrm{CM}$

Chromosome 19

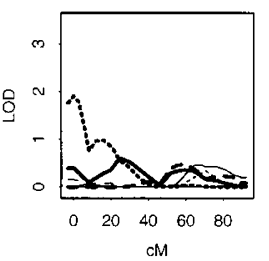

Chromosome 2

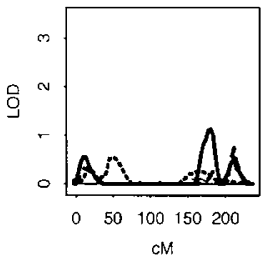

Chromosome 8

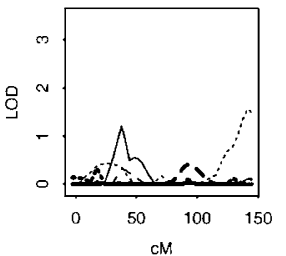

Chromosome 14

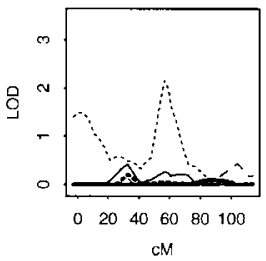

Chromosome 20

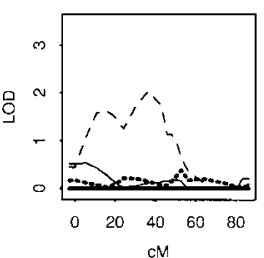

Chromosome 3

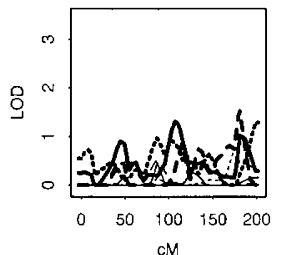

Chromosome 9

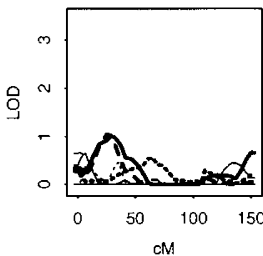

Chromosome 15

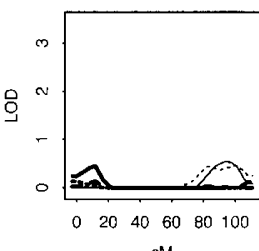

Chromosome 21

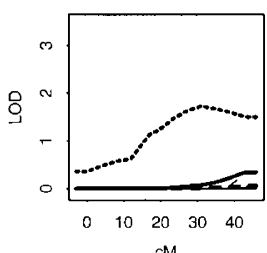

Chromosome 4

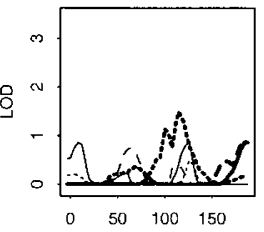

$\mathrm{cM}$

Chromosome 10

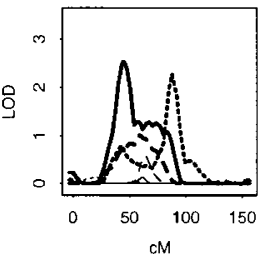

Chromosome 16

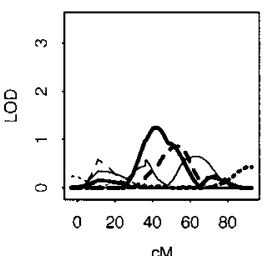

$\mathrm{CM}$

Chromosome 22

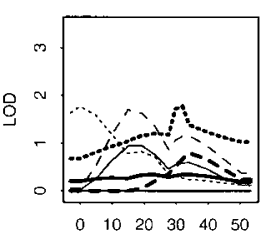

$\mathrm{CM}$
Chromosome 5

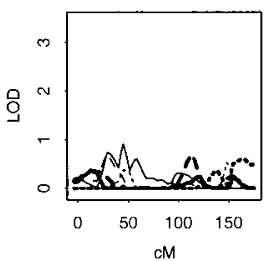

Chromosome 11

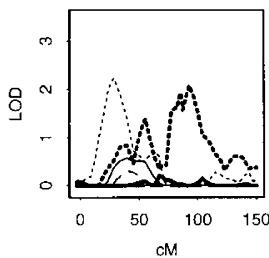

Chromosome 17

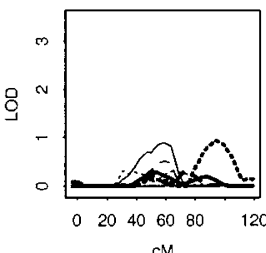

Chromosome $\mathrm{X}$

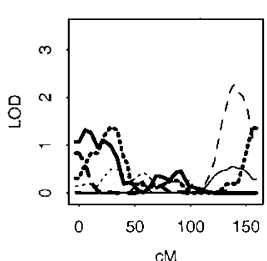

Chromosome 6

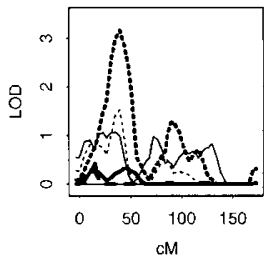

Chromosome 12

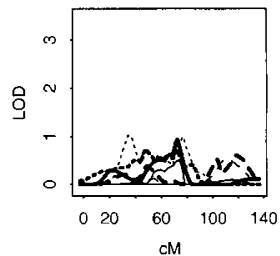

Chromosome 18
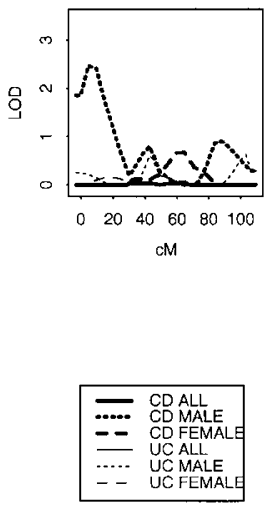

Figure 1 LOD scores for genome search family cohort stratified by sex of affected individuals, for CD and UC phenotypes.

LOD score occurred in male families on chromosome 1, with LOD scores of 2.13 in male NOD2 positive families $(n=13)$, and 0.07 in male NOD2 negative families $(n=8)$, compared with a total male LOD score of 2.76. This effect may, however, be due to the different numbers of families, rather than an epistatic effect between chromosome $1 \mathrm{q}$ and NOD2. We did not observe any difference in the frequency of NOD2 mutations between male and female cases with Crohn's disease (unpublished data).

\section{Discussion}

In this study, we have identified sex-specific linkage in both previously reported and novel candidate regions, with the highest evidence for linkage in the region of chromosome 6 surrounding the MHC region. Excess allele-sharing at this locus was observed in male-only affected families but not in female-only affected families, implying a disease gene in this region that increases susceptibility in males only. The role of
HLA genes in immune-mediated diseases is well established; a number of linkages of IBD to this region have been reported. ${ }^{10,25,26}$ HLA alleles are functional candidate loci for IBD susceptibility, playing a central role in the immune response. Several studies have demonstrated an association between HLA class II alleles and susceptibility to both CD and UC. $^{27,28}$ The TNF $\alpha$ locus is a functional and positional candidate gene for IBD, but association studies involving TNF $\alpha$ polymorphisms and IBD have given inconsistent results. ${ }^{10,29}$ The presence of sex-specific disease genes as well as allelic heterogeneity at a population level may partially explain the lack of replication in these studies. Differences in the frequency of HLA haplotypes between males and females occur in inflammatory bowel disease: DR3-DQ2 frequency was reduced in females with $\mathrm{UC}^{25}$ and $\mathrm{DRB} 1 * 15$ was increased only in female UC patients. ${ }^{30}$ Similar differences in HLA haplotype frequencies by sex occur in other immunemediated diseases including Type I diabetes, ${ }^{31}$ multiple sclerosis $^{32}$ and rheumatoid arthritis. ${ }^{33}$ 
Table 2 Details of linkage regions with maximum LOD score $>2$

\begin{tabular}{|c|c|c|c|c|c|c|c|c|}
\hline Chr & Region & $\begin{array}{c}c M \\
\text { from } \\
\text { pter }\end{array}$ & Phenotype & $A L L$ & $O D$ sco & $F$ & P-value & Markers \\
\hline 1 & $1 q 31-32$ & 186 & $C D$ & 0.16 & 2.76 & 0 & 0.0442 & $\begin{array}{l}\text { D1S413 } \\
\text { D1S249 }\end{array}$ \\
\hline 3 & $3 p 12$ & 87 & IBD & 0.85 & 2.05 & 0.05 & 0.1426 & D3S3653 \\
\hline 6 & $6 \mathrm{p} 22$ & 39 & $C D$ & 0.26 & 3.18 & 0 & 0.0024 & D6S276 \\
\hline 6 & $6 \mathrm{p} 22$ & 39 & IBD & 1.21 & 4.51 & 0 & $<0.0002$ & D6S276 \\
\hline 11 & $11 \mathrm{p} 15$ & 29 & UC & 0.38 & 2.24 & 0.07 & 0.0880 & D11S902 \\
\hline 14 & $14 q 23$ & 57 & UC & 0.27 & 2.17 & 0 & 0.0168 & D14S63 \\
\hline 18 & $18 \mathrm{p} 11$ & 5 & $C D$ & 0 & 2.46 & 0 & 0.0148 & $\begin{array}{l}\text { D18S59 } \\
\text { D18S452 }\end{array}$ \\
\hline 20 & $20 p 12-q 11$ & 36 & UC & 0.10 & 0 & 2.04 & 0.0536 & $\begin{array}{l}\text { D20S186 } \\
\text { D20S195 }\end{array}$ \\
\hline 22 & $22 q 13$ & 32 & IBD & 1.28 & 1.38 & 2.29 & 0.0010 & $\mathrm{D} 22 \mathrm{~S} 283$ \\
\hline
\end{tabular}

$\mathrm{M}=$ male-only affected families; $\mathrm{F}=$ female-only affected families; $\mathrm{ALL}=$ all families.

$P$-value is obtained using a permutation test for differences in LOD score between Male and Female families.

IBD

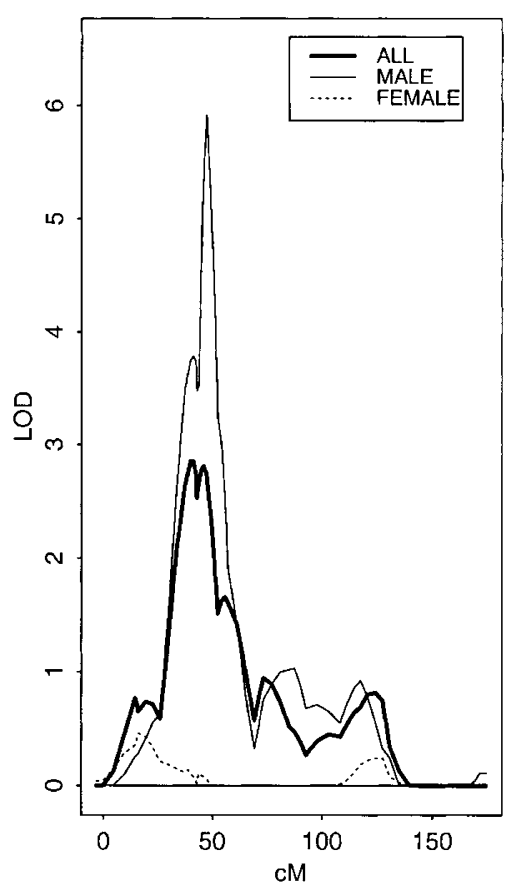

$\mathrm{CD}$

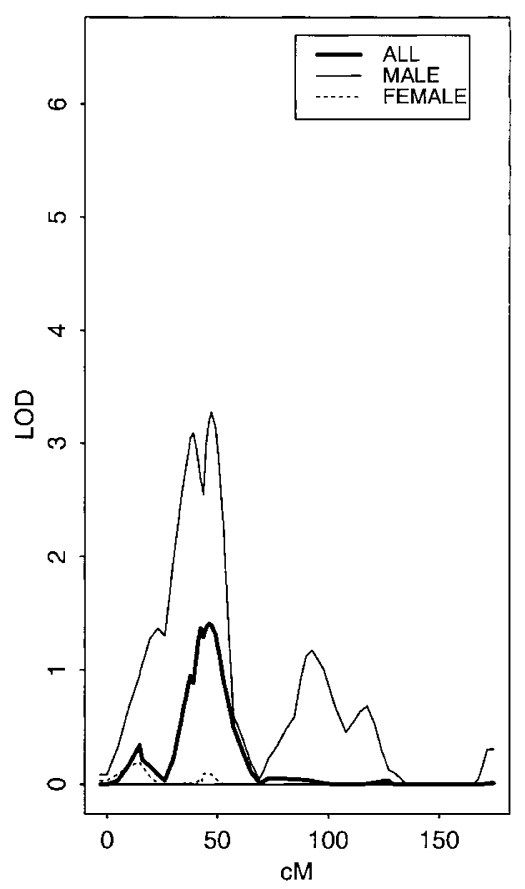

UC

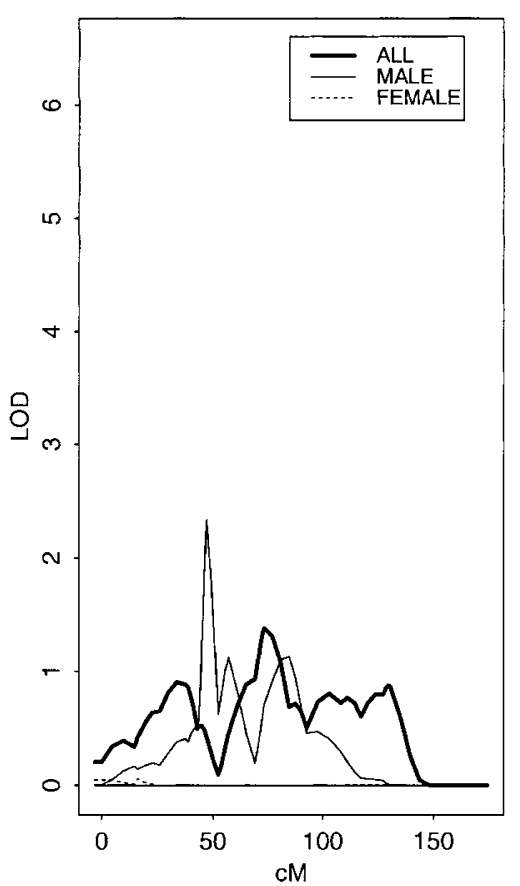

Figure 2 LOD scores on chromosome 6 for follow-up study family cohort stratified by sex of affected individuals, for IBD, CD and UC phenotypes.

Two other regions that showed strong evidence for malespecific linkage were chromosomes 1q31-32 and 18p11 (Table 2 and Figure 1). Neither of these regions was identified by genome scans. The established IBD candidate loci on chromosome 16 (IBD1) and chromosome 12 (IBD2) did not exhibit sex-specific linkage. Stratifying families by sex of affected individuals can substantially reduce the sample size to detect linkage. Few studies have tested the effect on power of stratifying affected sib pair families, but Leal and Ott ${ }^{34}$ showed that if excess identity-by-descent allele-sharing exists 
in a subgroup of sibpairs, then power to detect linkage is increased by stratification of the data. However, if no difference in linkage evidence between subgroups exists, stratification by phenotype will reduce power to detect linkage. The majority of the sex-specific linkages observed in this study occur mainly in male-only affected families, in contrast to the excess of female-only affected families for both CD and UC. Regions of male-only linkage would therefore have low power to be detected in the unstratified data, as shown by the low LOD scores for ALL families in Table 1. Our stratification analysis involves multiple testing across phenotypes and families in a genome-wide linkage analysis. No correction for multiple testing has been used, but a conservative Bonferroni correction for simulation tests identifies only chromosomes $6 \mathrm{p}$ and 22 as showing significant evidence for sex-specific linkage. Some of the results may therefore represent type I errors, and similar analyses in other IBD genome search linkage studies are necessary to establish which of these sex-specific linkages can be confirmed.

Sex effects in complex disease susceptibility have been reported in other human auto-immune disorders: female specific linkage to chromosomes 2, 6 and 11 was observed in osteoarthritis. ${ }^{20}$ Experimental animal models have also identified loci that exhibit sex-specific linkage in several complex diseases, for example in rheumatoid arthritis ${ }^{35}$ and Type I diabetes, ${ }^{36}$ although the molecular basis for such effects is unknown. It has been proposed that epigenetic factors play an important role in the pathogenesis of IBD, ${ }^{37}$ and that sex effects are mediated by androgens and oestrogens. Hormones have a substantial effect on gene expression, and the hormonal differences between males and females could thus lead to differential expression of disease susceptibility genes in males and females.

In conclusion, we have identified several putative regions of sex-specific linkage in IBD, including novel candidate loci. Most importantly, we have shown that evidence for linkage in the HLA region is restricted to families with only male affected individuals. This sex-specific linkage occurs in both CD and UC families. Stratification by sex or other phenotypic factors may reduce etiologic heterogeneity and lead to progress in the search for susceptibility loci in complex diseases.

\section{Acknowledgements}

This work was supported in the UK by the Wellcome Trust, the Generation Trust and Axys Pharmaceuticals Inc. In Germany, support was from the Deutsche Forschungsgemeinschaft (For423), a Training and Mobility of Research (TMR) Network grant of the European Union (ERB-4061-PL-97-0389), a Competence Network 'Chronisch-entzündliche Darmerkrankungen', the German Human Genome Project (DHGP) and the National Genome Research Network (all funded by the German Federal Department for Research and Education).

\section{References}

1 Hampe J, Cuthbert A, Croucher PJP et al: Association between insertion mutation in NOD2 gene and Crohn's disease in German and British populations. Lancet 2001; 357: 1925 - 1928.

2 Hugot JP, Chamaillard M, Zouali H et al: Association of NOD2 leucine-rich repeat variants with susceptibility to Crohn's disease. Nature 2001; 411: 599-603.

3 Ogura $\mathrm{Y}$, Bonen DK, Inohara $\mathrm{N}$ et al: A frameshift mutation in NOD2 associated with susceptibility to Crohn's disease. Nature 2001; 411: $603-606$.

4 Hugot JP, Laurent-Puig P, Gower-Rousseau C et al: Mapping of a susceptibility locus for Crohn's disease on chromosome 16. Nature 1996; 379: 821-823.

5 Satsangi J, Parkes M, Louis E et al: Two stage genome-wide search in inflammatory bowel disease provides evidence for susceptibility loci on chromosomes 3, 7 and 12. Nat Genet 1996; 14: $199-202$

6 Cho JH, Nicolae DL, Gold LH et al: Identification of novel susceptibility loci for inflammatory bowel disease on chromosomes 1p, 3q and 4q: evidence for epistatis between $1 \mathrm{p}$ and IBD1. Proc Natl Acad Sci USA 1998; 95: 7502-7507.

7 Ma Y, Ohmen JD, Li Z et al: A genome-wide search identifies potential new susceptibility loci for Crohn's disease. Inflam Bowel Dis 1999; 5: 271-278.

8 Duerr RH, Barmada MM, Zhang L, Pfützer R, Weeks DE: Highdensity genome scan in Crohn disease shows confirmed linkage to chromosome 14q11-12. Am J Hum Genet 2000; 66: 1857 1862.

9 Hampe J, Schreiber S, Shaw SH et al: A genomewide analysis provides evidence for novel linkages in inflammatory bowel disease in a large European cohort. Am J Hum Genet 1999; 64: $808-816$.

10 Hampe J, Shaw SH, Saiz R et al: Linkage of inflammatory bowel disease to human chromosome 6p. Am J Hum Genet 1999; 65: $1647-1655$.

11 Rioux JD, Silverberg MS, Daly MJ et al: Genomewide search in Canadian families with inflammatory bowel disease reveals two novel susceptibility loci. Am J Hum Genet 2000; 66: 1863 - 1870.

12 Forabosco P, Collins A, Latiano A et al: Combined segregation and linkage analysis of inflammatory bowel disease in the IBD1 region using severity to characterise Crohn's disease and ulcerative colitis. Eur J Hum Genet 2000; 8: 846-852.

13 Parkes M, Barmada MM, Satsangi J, Weeks DE, Jewell DP, Duerr RH: The IBD2 locus shows linkage heterogeneity between ulcerative colitis and Crohn's disease. Am J Hum Genet 2000; 67: $1605-1610$.

14 Brant SR, Panhuysen CIM, Bailey-Wilson JE et al: Linkage heterogeneity for the IBD1 locus in Crohn's disease pedigrees by disease onset and severity. Gastroenterology 2000; 119: 1483 1490.

15 de la Concha EG, Fernandez-Arquero M, Lopez-Nava G et al: Susceptibility to severe ulcerative colitis is associated with polymorphism in the central MHC gene IKBL. Gastroenterology 2000; 119: $1491-1495$.

16 Vermeire S, Satsangi J, Peeters $M$ et al: Evidence for inflammatory bowel disease of a susceptibility locus on the X chromosome. Gastroenterology 2001; 120: 834-840.

17 Ekbom A, Helmick C, Zack M, Adami HO: The epidemiology of inflammatory bowel disease: a large, population-based study in Sweden. Gastroenterology 1991; 100: 350-358.

18 Paterson AD, Petronis A: Age and sex based genetic locus heterogeneity in type 1 diabetes. J Med Genet 2000; 37: 186191.

19 O'Donnell CJ, Lindpaintner K, Larson MG et al: Evidence for association and genetic linkage of the angiotensin-converting enzyme locus with hypertension and blood pressure in men but not women in the Framingham Heart Study. Circulation 1998; 97: $1766-1772$. 
20 Loughlin J, Mustafa Z, Smith A et al: Linkage analysis of chromosome $2 \mathrm{q}$ in osteoarthritis. Rheumatology 2000; 39: $377-381$.

21 Wise LH, Lanchbury JS, Lewis CM: Meta-analysis of genome searches. Ann Hum Genet 1999: 63: 263-272.

22 Kong A, Cox NJ: Allele-sharing models: LOD scores and accurate linkage tests. Am J Hum Genet 1997; 61: 1179-1188.

23 Kruglyak L, Daly MJ, Reeve-Daly MP, Lander ES: Parametric and nonparametric linkage analysis: a unified multipoint approach Am J Hum Genet 1996; 58: 1347 - 1363.

24 Lander E, Kruglyak L: Genetic dissection of complex traits: guidelines for interpreting and reporting linkage results. Nat Genet 1995; 11: $241-247$.

25 Satsangi J, Welsh KI, Bunce M et al: Contribution of genes of the major histocompatibility complex to susceptibility and disease phenotype in inflammatory bowel disease. Lancet 1996; 347: $1212-1217$

26 Yang H, Plevy SE, Taylor K et al: Linkage of Crohn's disease to the major histocompatibility complex region is detected by multiple non-parametric analyses. Gut 1999; 44: 519-526.

27 Kawasaki A, Tsuchiya N, Hagiwara K, Takazoe M, Tokunaga K Independent contribution of HLA-DRB1 and TNF alpha promoter polymorphisms to the susceptibility to Crohn's disease. Genes Immun 2000; 1: 351 - 357.

28 Trachtenberg EA, Yang H, Hayes E et al: HLA class II haplotype associations with inflammatory bowel disease in Jewish (Ashkenazi) and non-Jewish caucasian populations. Hum Immunol 2000; 61: 326-333.

29 Koss K, Satsangi J, Fanning GC, Welsh KI, Jewell DP: Cytokine (TNF alpha, LT alpha and IL-10) polymorphisms in inflammatory bowel diseases and normal controls: differential effects on production and allele frequencies. Genes Immun 2000; 1: 185 190.
30 Bouma G, Oudkerk Pool M et al: Evidence for genetic heterogeneity in inflammatory bowel disease (IBD); HLA genes in the predisposition to suffer from ulcerative colitis (UC) and Crohn's disease (CD). Clin Exp Immunol 1997; 109: 175 - 179.

31 Cucca F, Goy JV, Kawaguchi Y et al: A male-female bias in type 1 diabetes and linkage to chromosome Xp in MHC HLA-DR3positive patients. Nat Genet 1998; 19: 301-302.

32 Celius EG, Harbo HF, Egeland T, Vartdal F, Vandvik B, Spurkiand A: Sex and age at diagnosis are correlated with the HLADR2,DQ6 haplotype in multiple sclerosis. J Neurol Sci 2000; 178: $132-135$.

33 Hajeer A, John S, Ollier WE et al: Tumor necrosis factor microsatellite haplotypes are different in male and female patients with RA. J Rheumatol 1997; 24: $217-219$.

34 Leal SM, Ott J: Effects of stratification in the analysis of affected sib-pair data: benefits and costs. Am J Hum Genet 2000; 66: 567 575.

35 Vingsbo-Lundberg C, Nordquist N, Olofsson P et al: Genetic control of arthritis onset, severity and chronicity in a model for rheumatoid arthritis in rats. Nat Genet 1998; 20: $401-404$.

36 Melanitou E, Joly F, Lathrop M, Boitard C, Avner P: Evidence for the presence of insulin-dependent diabetes-associated alleles on the distal part of mouse chromosomes 6. Genome Res 1998; 8: $608-620$.

37 Petronis A, Petroniene R: Epigenetics of inflammatory bowel disease. Gut 2000; 47: 302-306. 